\title{
Aqueous Extract of Paeonia lactiflora and Paeoniflorin as Aggregation Reducers Targeting Chaperones in Cell Models of Spinocerebellar Ataxia 3
}

\author{
Kuo-Hsuan Chang, ${ }^{1}$ Wan-Ling Chen, ${ }^{1}$ Li-Ching Lee, ${ }^{2}$ Chih-Hsin Lin, ${ }^{1}$ \\ Pin-Jui Kung, ${ }^{2}$ Te-Hsien Lin, ${ }^{2}$ Yi-Ci Wu, ${ }^{2}$ Yih-Ru Wu, ${ }^{1}$ Yi-Chun Chen, ${ }^{1}$ \\ Guey-Jen Lee-Chen, ${ }^{2}$ and Chiung-Mei Chen ${ }^{1}$ \\ ${ }^{1}$ Department of Neurology, Chang Gung Memorial Hospital, Chang Gung University College of Medicine, Taipei 10507, Taiwan \\ ${ }^{2}$ Department of Life Science, National Taiwan Normal University, Taipei 11677, Taiwan
}

Correspondence should be addressed to Guey-Jen Lee-Chen; t43019@ntnu.edu.tw

and Chiung-Mei Chen; cmchen@adm.cgmh.org.tw

Received 29 October 2012; Accepted 19 January 2013

Academic Editor: Carlo Ventura

Copyright ( 2013 Kuo-Hsuan Chang et al. This is an open access article distributed under the Creative Commons Attribution License, which permits unrestricted use, distribution, and reproduction in any medium, provided the original work is properly cited.

\begin{abstract}
Spinocerebellar ataxia (SCA) types 1, 2, 3, 6, 7, and 17 as well as Huntington's disease are a group of neurodegenerative disorders caused by expanded CAG repeats encoding a long polyglutamine (polyQ) tract in the respective proteins. Evidence has shown that the accumulation of intranuclear and cytoplasmic misfolded polyQ proteins leads to apoptosis and cell death. Thus suppression of aggregate formation is expected to inhibit a wide range of downstream pathogenic events in polyQ diseases. In this study, we established a high-throughput aggregation screening system using 293 ATXN3/ ${ }_{75}$-GFP cells and applied this system to test the aqueous extract of Paeonia lactiflora (P. lactiflora) and its constituents. We found that the aggregation can be significantly prohibited by P. lactiflora and its active compound paeoniflorin. Meanwhile, P. lactiflora and paeoniflorin upregulated HSF1 and HSP70 chaperones in the same cell models. Both of them further reduced the aggregation in neuronal differentiated SH-SY5Y ATXN3/ ${ }_{75}{ }^{-}$ GFP cells. Our results demonstrate how P. lactiflora and paeoniflorin are likely to work on polyQ-aggregation reduction and provide insight into the possible working mechanism of P. lactiflora in SCA3. We anticipate our paper to be a starting point for screening more potential herbs for the treatment of SCA3 and other polyQ diseases.
\end{abstract}

\section{Introduction}

Spinocerebellar ataxias (SCAs) are a large, complex group of heterogeneous autosomal dominant neurodegenerative disorders characterized by cerebellar dysfunction alone or in combination with other neurological abnormalities [1]. Among them, the expansions of CAG trinucleotide repeats encoding a polyglutamine (polyQ) stretch have been shown to cause dominantly inherited SCA1, SCA2, SCA3, SCA6, SCA7, SCA17, and dentatorubropallidoluysianatrophy (DRPLA) [2-8]. These polyQ-mediated genetic disorders in SCAs have shown selective progressive degeneration of the cerebellum, brainstem, and spinal tract, with prominent pathological hallmark of intranuclear and cytoplasmic accumulation of aggregated polyQ proteins inside degenerated neurons [9]. Different polyQ tractcontaining proteins ultimately lead to the dysfunction and degeneration of specific neuronal subpopulations [10]. The aggregated polyQ proteins may cause dysfunction of mitochondria, chaperone, and ubiquitin proteasome system, leading to apoptosis and cell death [11-13]. As misfolding of the polyQ protein is likely the initial event in the pathogenic cascade, suppression of protein misfolding is expected to inhibit a wide range of downstream detrimental events, and to rescue neuronal dysfunction.

Increasing evidence suggests that some herbs may potentially attenuate the deterioration of neurodegenerative diseases. Paeonia lactiflora (P. lactiflora), belonging to the 
Paeoniaceae family, is a perennial herb frequently used as an important ingredient in many traditional prescriptions. It has been commonly used for nourishing blood, alleviating pain, reducing irritability, as well as treating liver disease and cancer [14]. Paeoniflorin, one of the main compounds extracted from $P$. lactiflora, has been reported to ameliorate neurodegenerative process in Parkinson's disease (PD) and Alzheimer's disease (AD) models [15-17]. However, the effect of $P$. lactiflora herb extract and paeoniflorin in treating SCA remains unraveled.

In the present study, we firstly built up an aggregation screening cell model by overexpressing CAG-expanded ATXN3, the causative mutation in SCA3 [2], in 293 cells, and then examined the anti-aggregation effect of $P$. lactiflora aqueous extract and paeoniflorin. We further demonstrated that the anti-aggregation activity of P. lactiflora extract and paeoniflorin was contributed by the enhancement of heat shock transcription factor 1 (HSF1)-heat shock protein (HSP) 70 chaperone system. These findings provide evidence that P. lactiflora and paeoniflorin may be a novel alternative therapeutic agent for the treatment of SCAs.

\section{Materials and Methods}

2.1. P. lactiflora Extract Preparation and HPLC Analysis. Aqueous extract from $P$. lactiflora was provided by SunTen Pharmaceutical Company (Taipei, Taiwan). Briefly, $100 \mathrm{~g}$ of dried P. lactiflora was boiled with $1500 \mathrm{~mL}$ of water at $100^{\circ} \mathrm{C}$ for $30 \mathrm{~min}$ and was sieved using a 100-mesh sieve. The extract was concentrated to $100 \mathrm{~mL}$ and filtered through a 200-mesh sieve. The extract was then dried by speed vacuum concentration and then stored at $-20^{\circ} \mathrm{C}$ until used.

High pressure liquid chromatography (HPLC) was performed using a LaChrom Elite HPLC system (Hitachi), consisting of a photo diode array detector. The chromatographic separation of $P$. lactiflora extract $(50 \mu \mathrm{L}, 1 \mathrm{mg} / \mathrm{mL})$ was carried out on a Hypersil ODS (C18) column $(250 \times 4.6 \mathrm{~mm}, 5 \mu \mathrm{m})$, eluted with the mixture of $0.1 \%$ formic acid in water (A) or acetonitrile (B). The linear gradient elution program for $\mathrm{A}: \mathrm{B}(\mathrm{v} / \mathrm{v})$ was set as follows: $95: 5(0-10 \mathrm{~min}), 95: 5-70: 30$ (10-40 min), 70:30-15:85 (40-55 min), 15:85-95:5 (55$60 \mathrm{~min}), 95: 5(60-75 \mathrm{~min})$ with a flow rate of $1 \mathrm{~mL} / \mathrm{min}$. Absorbance was monitored at 230, 250, $270 \mathrm{~nm}$ and the scan range for photo diode array was $190 \sim 400 \mathrm{~nm}$. Paeoniflorin, gallic acid, and albiflorin $(2 \sim 10 \mu \mathrm{L}, 20 \mathrm{mM})$ were used as reference compounds for P. lactiflora $[18,19]$.

2.2. Cell Culture and Cell Proliferation Assay. Human embryonic kidney HEK-293 cells (ATCC No. CRL-1573) were cultivated in Dulbecco's modified Eagle's medium (DMEM) containing $10 \%$ fetal bovine serum (FBS). Human neuroblastoma SH-SY5Y cells (ATCC No. CRL-2266) were maintained in DMEM F12 supplemented with 10\% FBS. Cells were cultivated at $37^{\circ} \mathrm{C}$ incubator containing $5 \% \mathrm{CO}_{2}$ and cell proliferation was measured based upon the reduction of the tetrazolium salt, 3,[4,5-dimethylthiazol-2-yL]-2,5-diphenyltetrazolium bromide (MTT). Cells were plated into 48well $\left(5 \times 10^{4} /\right.$ well $)$ dishes, grown for $20 \mathrm{hr}$ and treated with different concentrations of the P. lactiflora extract (5 $30 \mathrm{mg} / \mathrm{mL})$ or pure compound $(100 \mathrm{nM} 1 \mathrm{mM})$. After one day, $20 \mu \mathrm{L}$ MTT $(5 \mathrm{mg} / \mathrm{mL}$ in PBS, Sigma) was added to cells and incubated for $2 \mathrm{hr}$. The absorbance of the purple formazan dye was measured at $570 \mathrm{~nm}$ by a Bio-Tek $\mu$ Quant Universal Microplate Spectrophotometer.

2.3. Flp-In-293 Triple Fluorescent Reporter Cells and Fluorescent Assay. A triple fluorescent reporter plasmid with mCherry, ZsYellow1, and AmCyan1 was first constructed in pAmCyan1-N1. A proximal promoter $(-360 \sim+2$, with the translation initiation site as +1 ) from HSF1 gene was used, as $331 \mathrm{bp}$ upstream of the murine HSF1 translation start site is required for maximal basal expression [20]. Promoter fragments from HSF1 (enhancing chaperone expression), heat shock cognate protein (HSPA8, -1140 +38 , driving constitutively expressed HSP70) [21] and heatinducible HSP70 chaperone (HSPA1A, -273 +215, driving heat-inducible HSP70) [22] are placed upstream of the three fluorescent reporters. The fragment containing the HSF1, HSPA8, and HSPA1A driven reporters was excised with AseI and NotI restriction enzymes and used to replace an AseI-NotI fragment in pcDNA5/FRT/TO plasmid (Invitrogen). The resulting triple fluorescent reporter plasmid was used to generate Flp-In triple fluorescent reporter cells and maintained according to the supplier's instructions (Invitrogen). Geranylgeranylacetone (GGA, Sigma) or paeoniflorin $(100 \mathrm{nM} 100 \mu \mathrm{M})$ was added to the medium for $24 \mathrm{hr}$. The three fluorescence colors were analyzed simultaneously using high-content analysis (HCA) system (ImageXpressMICRO, Molecular Devices), with excitation/emission wavelengths at 453/486 (mCherry), 531/540 (ZsYellow1) and 587/610 nm (AmCyan1).

2.4. ATXN3 and HSF1 cDNA Constructs. Polyadenylated RNA (200 ng) isolated from neuroblastoma SK-N-SH cells was reverse transcribed using the SuperScript III reverse transcriptase (Invitrogen). The sense and antisense primers used for ATXN3/Q 14 cDNA (+826 +1152, NM_004993) amplification were 5'-ATTCAGCTAAGTATGCAAGGTAGTTCCA (codon for Met257 underlined) and 5'-CATGCCATGGCATGTTTTTTTCCTTCTGTT (NcoI site underlined). The amplified $3^{\prime}$ polyQ-containing cDNA fragment (translated into amino acids 257 361) was cloned into pGEM-T Easy (Promega) and sequenced. The ATXN3/ $Q_{14}$ cDNA was excised with EcoRI (in pGEM-T Easy vector) and $\mathrm{NcoI}$ and subcloned into pEGFP-N1 (Clontech). Then DNA fragment containing in-frame $A T X N 3 / \mathrm{Q}_{14}{ }^{-}$ EGFP was excised with HindIII-NotI and subcloned into the pcDNA5/FRT/TO. The ATXN3/Q ${ }_{75}$ cDNA was made by replacing an $88 \mathrm{bp}$ ATXN3/ $\mathrm{Q}_{14} B s m \mathrm{BI}-B s m \mathrm{FI}$ fragment with a $271 \mathrm{bp}$ ATXN3/ $\mathrm{Q}_{75}$ fragment from the cDNA clone of a SCA3 patient. The HSF1 cDNA (BC014638) in pOTB7 was obtained from Bioresource Collection and Research Center (BCRC), Food Industry Research and Development Institute, Taiwan. The cDNA was excised with EcoRI and XhoI and subcloned into pcDNA3 (Invitrogen). 


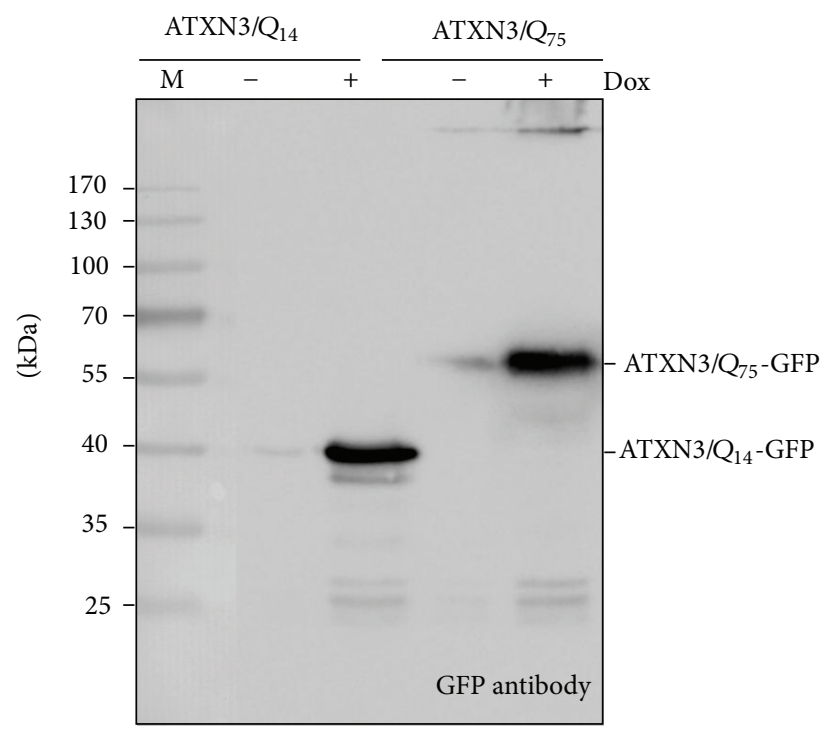

(a)

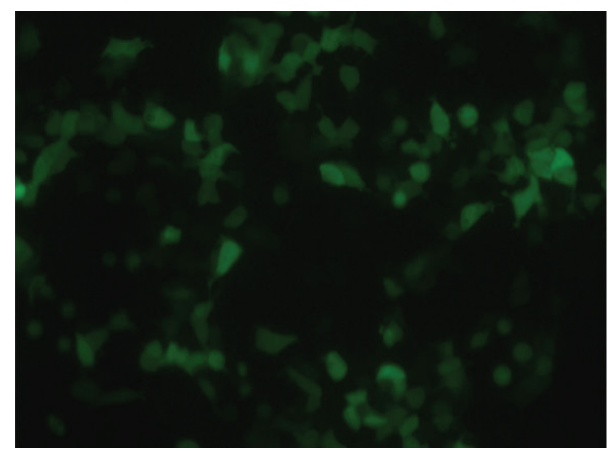

ATXN3/Q $14-G F P$

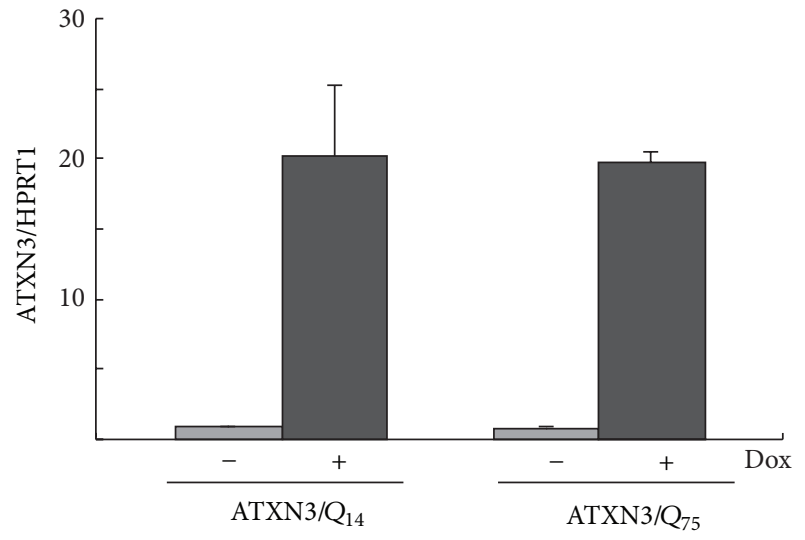

(b)

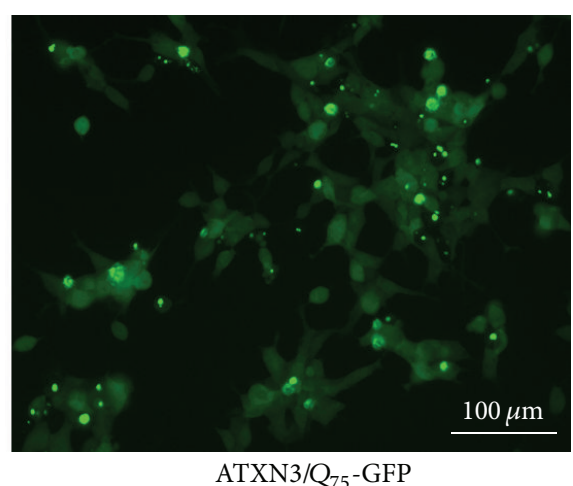

ATXN3/Q $75-G F P$

(c)

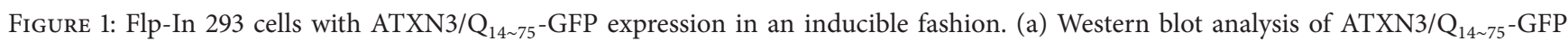
protein expression using GFP antibody after two days of induction (+Dox). (b) Real-time PCR quantification of $\mathrm{ATXN} / \mathrm{Q}_{14 \sim 75}-\mathrm{GFP}$ RNA expression relatively to HPRT after two days of induction (+Dox). (c) Fluorescence microscopy images of $A T X N 3 / Q_{14 \sim 75}-\mathrm{GFP}_{\text {expression }}$ after six days of induction. The scale bar $=100 \mu \mathrm{m}$.

2.5. Isogenic 293 and SH-SY5Y Cell Lines. Human 293derived Flp-In-293 cells (Invitrogen) were cultivated in DMEM containing $10 \% \mathrm{FBS}$ as described. The cloned pcDNA5/FRT/TO-ATXN3/ $\mathrm{Q}_{14}$ and $\mathrm{Q}_{75}$ plasmids were used to generate the isogenic $A T X N 3 / Q_{14 \sim 75}$ cell lines by targeting insertion into Flp-In-293 cells, according to the supplier's instructions. The repeats in these ATXN3 cell lines were examined by PCR and sequencing. These cell lines were grown in medium containing $5 \mu \mathrm{g} / \mathrm{mL}$ blasticidin and $100 \mu \mathrm{g} / \mathrm{mL}$ hygromycin (InvivoGen). Human SH-SY5Yderived Flp-In host cell line was constructed as described [23]. The SH-SY5Y host cells were used to generate isogenic ATXN3/ $Q_{14 \sim 75}$ lines and maintained as described above.

2.6. ATXN3/Q $\mathrm{Q}_{75}$ Aggregation Assay. 293ATXN3/Q 75 -GFP cells were plated into 96 -well $\left(2 \times 10^{4} /\right.$ well $)$ dishes, grown for $24 \mathrm{hr}$ and treated with different concentrations of the
P. lactiflora extract $(2 \sim 200 \mu \mathrm{g} / \mathrm{mL})$ or suberoylanilide hydroxamic acid (SAHA, Cayman Chemical), paeoniflorin (Sigma), gallic acid, and albiflorin (Chromadex) (100 nM 5 $\mu \mathrm{M})$ for $8 \mathrm{hr}$. Then doxycycline $(10 \mu \mathrm{g} / \mathrm{mL}, \mathrm{BD})$ was added to the medium to induce ATXN3/Q ${ }_{75}$-GFP expression for 6 days. Oxaliplatin ( $5 \mu \mathrm{M}$, Sigma) was also added for aggregate accumulation through inhibition of cell division [24]. Then cells were stained with Hochest $33342(0.1 \mu \mathrm{g} / \mathrm{mL}$, Sigma $)$ and aggregation percentage was assessed by HCA system, with excitation/emission wavelengths at 482/536 (GFP).

SH-SY5Y ATXN3/ ${ }_{75}$-GFP cells were seeded in 6-well $\left(2 \times 10^{5} /\right.$ well $)$ plate, with all trans retinoic acid $(10 \mu \mathrm{M}$, Sigma) added at seeding time. At day 2, cells were treated with paeoniflorin $(100 \mathrm{nM})$ or the P. lactiflora extract $(10 \mu \mathrm{g} / \mathrm{mL})$ for $8 \mathrm{hr}$, and then doxycycline $(5 \mu \mathrm{g} / \mathrm{mL})$ was added to induce ATXN3/ $Q_{75}$ GFP expression. The cells were kept in the medium containing $10 \mu \mathrm{M}$ trans retinoic acid, doxycycline and paeoniflorin/P. lactiflora extract for 7 days. After that, 


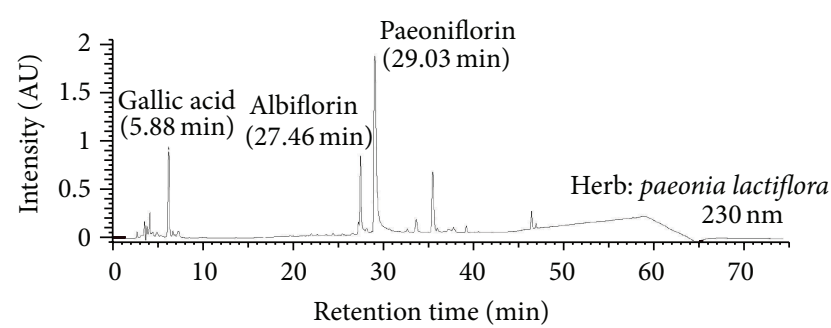

(a)

\begin{tabular}{|l|c|r|}
\hline & \multicolumn{2}{|c|}{ Content in $1 \mathrm{~g} / \mathrm{mL}$} \\
\hline Paeoniflorin & $2.27 \%$ & $47.33 \mathrm{mM}$ \\
\hline Gallic acid & $0.3 \%$ & $18.06 \mathrm{mM}$ \\
\hline Albiflorin & $0.73 \%$ & $15.16 \mathrm{mM}$ \\
\hline
\end{tabular}

(b)

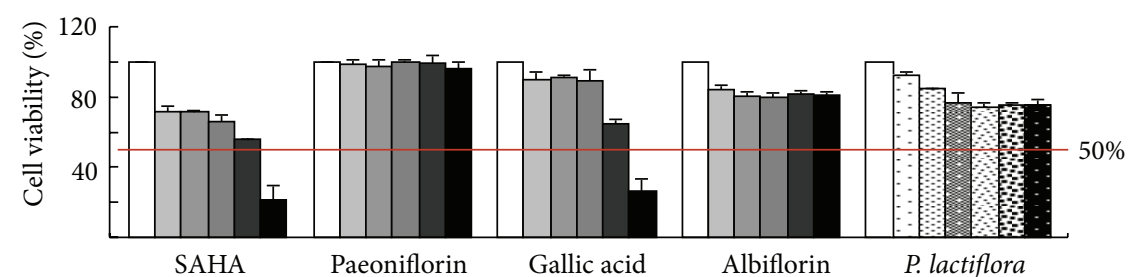

HEK-293 IC 50 cytotoxicity: $0.38 \mathrm{mM} \quad>1 \mathrm{mM} \quad>.58 \mathrm{mM} \quad>1 \mathrm{mM} \quad>30 \mathrm{mg} / \mathrm{mL}$

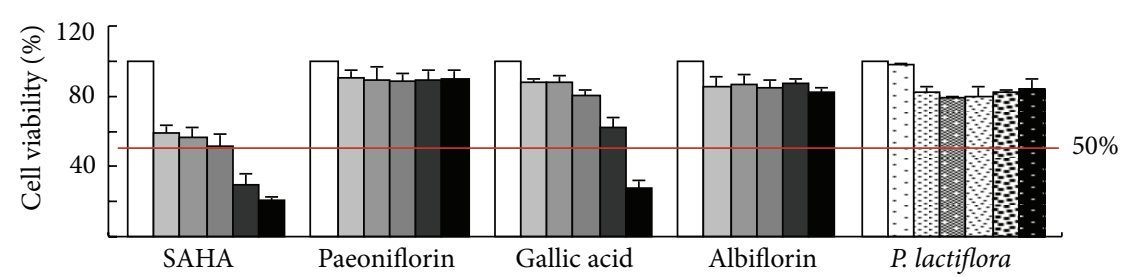

SH-SY5Y IC 50 cytotoxicity:

$\begin{array}{lcccc}0.02 \mathrm{mM} & >1 \mathrm{mM} & 0.57 \mathrm{mM} & >1 \mathrm{mM} & >30 \mathrm{mg} / \mathrm{mL} \\ & & & & \\ \text { Untreated } & 10 \mu \mathrm{M} & \ddots & 5 \mathrm{mg} / \mathrm{mL} & 20 \mathrm{mg} / \mathrm{mL} \\ 100 \mathrm{nM} & 100 \mu \mathrm{M} & 1 \mathrm{mM} & 10 \mathrm{mg} / \mathrm{mL} & 25 \mathrm{mg} / \mathrm{mL} \\ 1 \mu \mathrm{M} & & 15 \mathrm{mg} / \mathrm{mL} & \because & 30 \mathrm{mg} / \mathrm{mL}\end{array}$

(c)

FIgure 2: Chemical profile and cytotoxicity of the aqueous extract of $P$. lactiflora. (a) Chromatographic patterns from HPLC analysis $(230 \mathrm{~nm})$ showed peaks compatible with paeoniflorin, albiflorin and garlic acid. (b) The relative amount of above molecules in the extract. (c) Cytotoxicity of the aqueous extract of P. lactiflora, paeoniflorin, garlic acid, albiflorin, and SAHA against HEK-293 and SH-SY5Y cells using MTT viability assay. The $\mathrm{IC}_{50}$ of each herb/compound was shown under the columns. To normalize, the relative viability in untreated cells is set as $100 \%$. The red line represents $50 \%$ viability.

cells were stained with Hochest $33342(0.1 \mu \mathrm{g} / \mathrm{mL})$ and aggregation percentage was assessed as described.

2.7. Real-Time PCR. Total RNA from 293 ATXN3 lines was extracted using Trizol reagent (Invitrogen). The RNA was DNase (Stratagene) treated, quantified, and reversetranscribed to cDNA as described. Real-time quantitative PCR experiments were performed in the ABI PRISM 7000 Sequence Detection System (Applied Biosystems). Amplification was performed on $100 \mathrm{ng}$ cDNA with genespecific TaqMan fluorogenic probes Hs0024525_ml for ATXN3 and 4326321E for HPRT1 (endogenous control) (Applied Biosystems). Fold change was calculated using the formula $2^{\Delta \mathrm{C} t}, \Delta \mathrm{C}_{t}=\mathrm{C}_{t}$ (control) $-\mathrm{C}_{t}$ (target), in which $\mathrm{C}_{t}$ indicates cycle threshold.

2.8. Western Blot Analysis. Total proteins were prepared using lysis buffer containing $50 \mathrm{mM}$ Tris- $\mathrm{HCl}, 150 \mathrm{mM} \mathrm{NaCl}$,
$1 \mathrm{mM}$ EDTA, $1 \mathrm{mM}$ EGTA, $0.1 \%$ SDS and $0.5 \%$ sodium deoxycholate, $1 \%$ Triton X-100, protease inhibitor cocktail (Calbiochem). Proteins ( $25 \mu \mathrm{g}$ ) were separated on $10 \%$ SDSpolyacrylamide gel electrophoresis and blotted on to nitrocellulose membranes by reverse electrophoresis. After blocking, the membrane was probed with HSF1 (1:1000 dilution, Abnova), HSPA8 (1:500 dilution, Santa Cruz), HSPA1A (1:500 dilution, Santa Cruz), H3F3B (1:3000 dilution, GeneTex), GFP (1:500 dilution, Santa Cruz), $\beta$-actin (1:5000 dilution, Millipore) or GAPDH (1:1000 dilution, MDBio) at $4^{\circ} \mathrm{C}$ overnight. Then the immune complexes were detected by horseradish peroxidase-conjugated goat anti-mouse or goat anti-rabbit IgG antibody (1:5000 dilution, GeneTex) and chemiluminescent substrate (Millipore).

2.9. ATXN3/Q $\mathrm{Q}_{75}$ and HSF1 cDNA Co-Transfection. Human embryonic kidney HEK-293T cells (ATCC No. CRL-11268) were cultivated in DMEM containing 10\% FBS as described. 


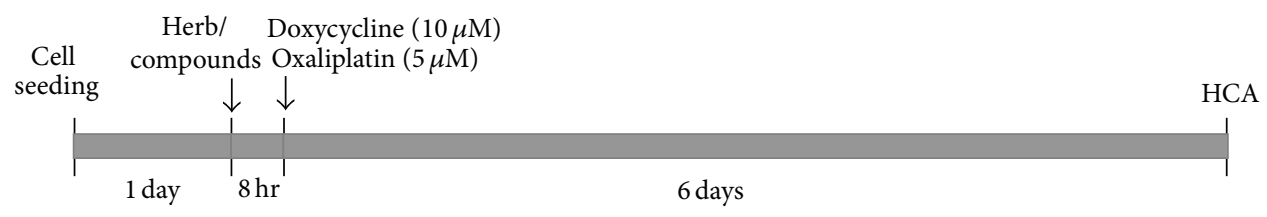

(a)
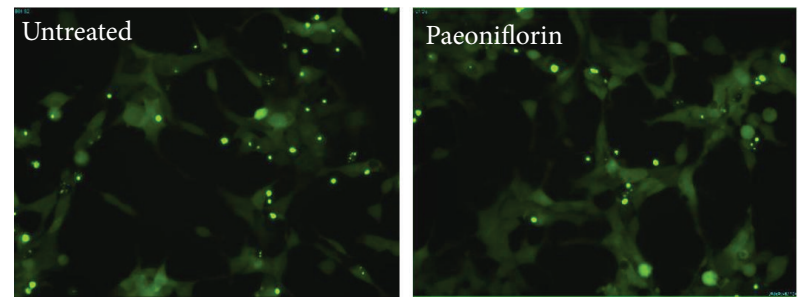

(b)

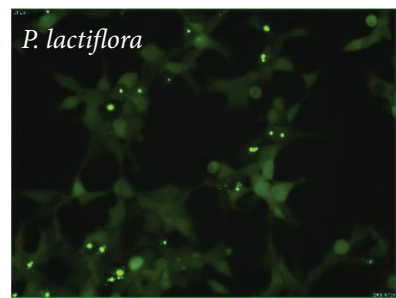

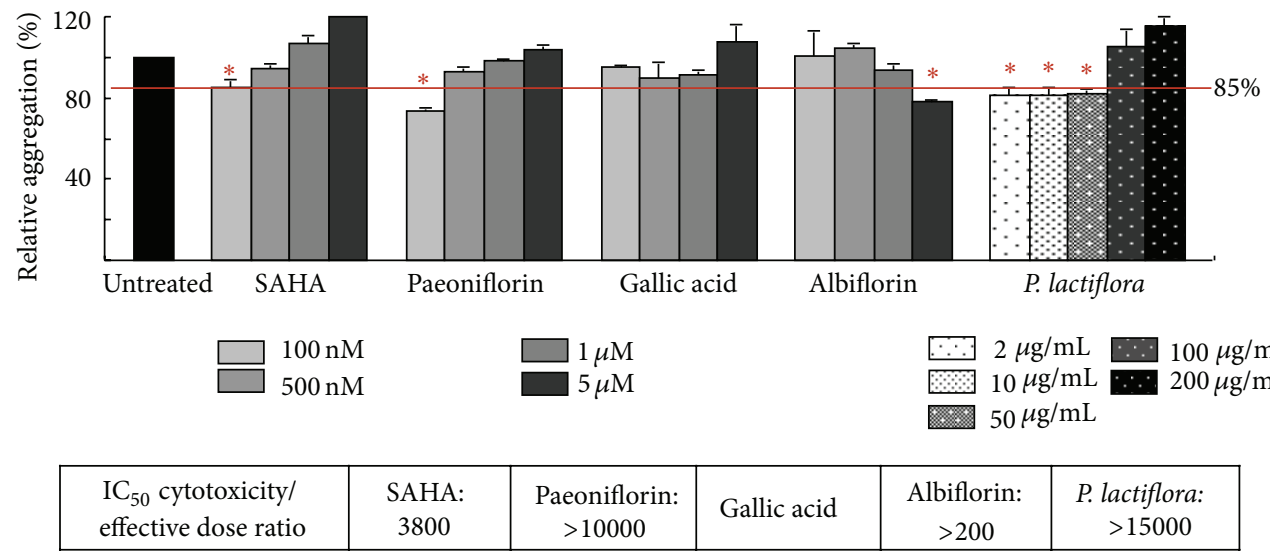

(c)

FIGURE 3: High-content compound screen using Flp-In 293 cells with inducible ATXN3/Q ${ }_{75}$-GFP expression. (a) Experiment flow chart. ATXN3/Q ${ }_{75}$-GFP 293 cells were plated into 96-well dishes, grown for $24 \mathrm{hr}$ and treated with different concentrations of the herb or compound for $8 \mathrm{hr}$. Then doxycycline and oxaliplatin was added to the medium to for 6 days and aggregation percentage was assessed by HCA system. (b) Representative fluorescence microscopy images of ATXN3/ $\mathrm{Q}_{75}$-GFP cells untreated or treated with P. lactiflora $(10 \mu \mathrm{g} / \mathrm{mL})$ or paeoniflorin $(100 \mathrm{nM})$ for 6 days. (c) Aggregation analysis of ATXN3/ $\mathrm{Q}_{75}$-GFP cells untreated or treated with aqueous extract of $P$. lactiflora $(2 \sim 200 \mu \mathrm{g} / \mathrm{mL})$, paeoniflorin, garlic acid, albiflorin and SAHA $(100 \mathrm{nM} \sim 5 \mu \mathrm{M})$. To normalize, the relative aggregation level in untreated cells is set as $100 \%$. The red line represents $85 \%$ aggregation with SAHA treatment $(100 \mathrm{nM})$.

For transient overexpression, cells were plated into 12well $\left(1 \times 10^{5} /\right.$ well $)$ dishes, grown for $20 \mathrm{hr}$, and transfected using T-Pro reagent (JF Biotechnology, Taiwan) with pcDNA5/FRT/TO-ATXN3/ ${ }_{75}$ and pcDNA3-HSF1 or pcDNA3 vector plasmids (1.5 $\mu \mathrm{g}$ each). The cells were grown for $48 \mathrm{hr}$ for ATXN3/ $\mathrm{Q}_{75}$ aggregation assay as described.

2.10. Statistical Analysis. For each set of values, data were expressed as the means \pm standard deviation (SD). Three independent experiments were performed and non-categorical variables were compared using the Student's $t$-test. All $P$ values were two-tailed, with values of $P<0.05$ considered significant.

\section{Results}

3.1. Construction of 293 Cells Expressing ATXN3/Q ${ }_{75}$ Aggregates. For therapies toward the polyQ diseases, we aimed to screen herbs/compounds potentially inhibiting polyQ aggregation. As removal of the N-terminus of polyQ-expanded ATXN3 is required for aggregation in vitro and in vivo [25], we cloned GFP-tagged ATXN3 C-terminal $\mathrm{Q}_{14 \sim 75}$-containing fragment to establish Flp-In 293 cells with ATXN3/ $\mathrm{Q}_{14 \sim 75^{-}}$ GFP expression in an inducible fashion. As shown in Figure 1(a), the GFP antibody detected $40 \mathrm{kDa} A T X N 3 / \mathrm{Q}_{14}{ }^{-}$ GFP and $57 \mathrm{kDa} A T X N 3 / \mathrm{Q}_{75}$-GFP proteins in doxycycline (Dox) induced ATXN3 cells. ATXN3-RNA levels were then examined by real-time PCR using ATXN3-specific probe and primers. As shown in Figure 1(b), in the presence of Dox, the two ATXN3 lines expressed 20 times more ATXN3 RNA than in the absence of Dox. While the expressed ATXN3/ $\mathrm{Q}_{14}$ was mainly diffused, the expressed ATXN3/ $\mathrm{Q}_{75}$-GFP formed aggregates (Figure 1(c)).

3.2. Aqueous Extract of P. lactiflori and Constituents. To examine the potential active compounds in P. lactiflori, 


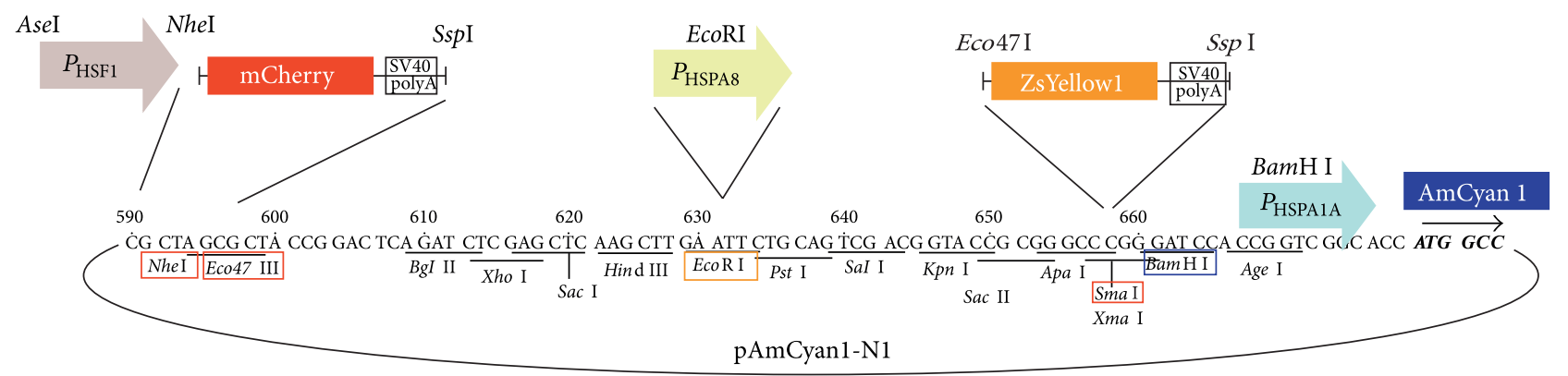

(a)

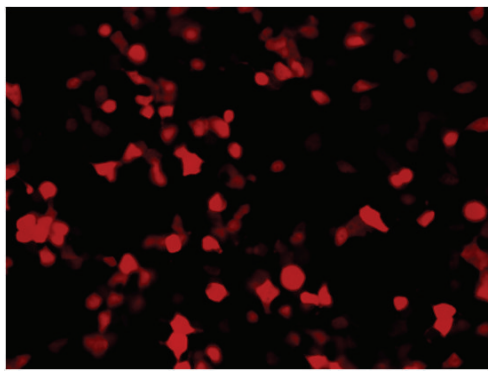

mCherry

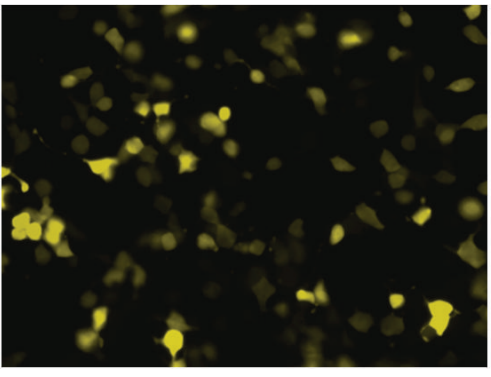

Yellow1

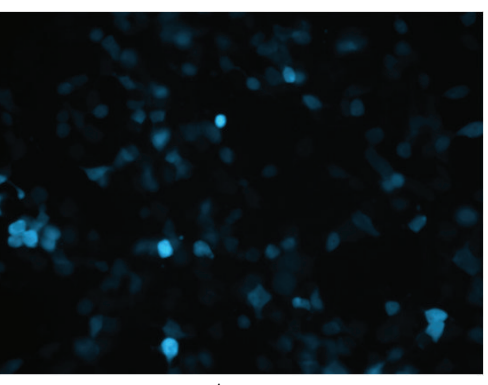

Amcyan

(b)

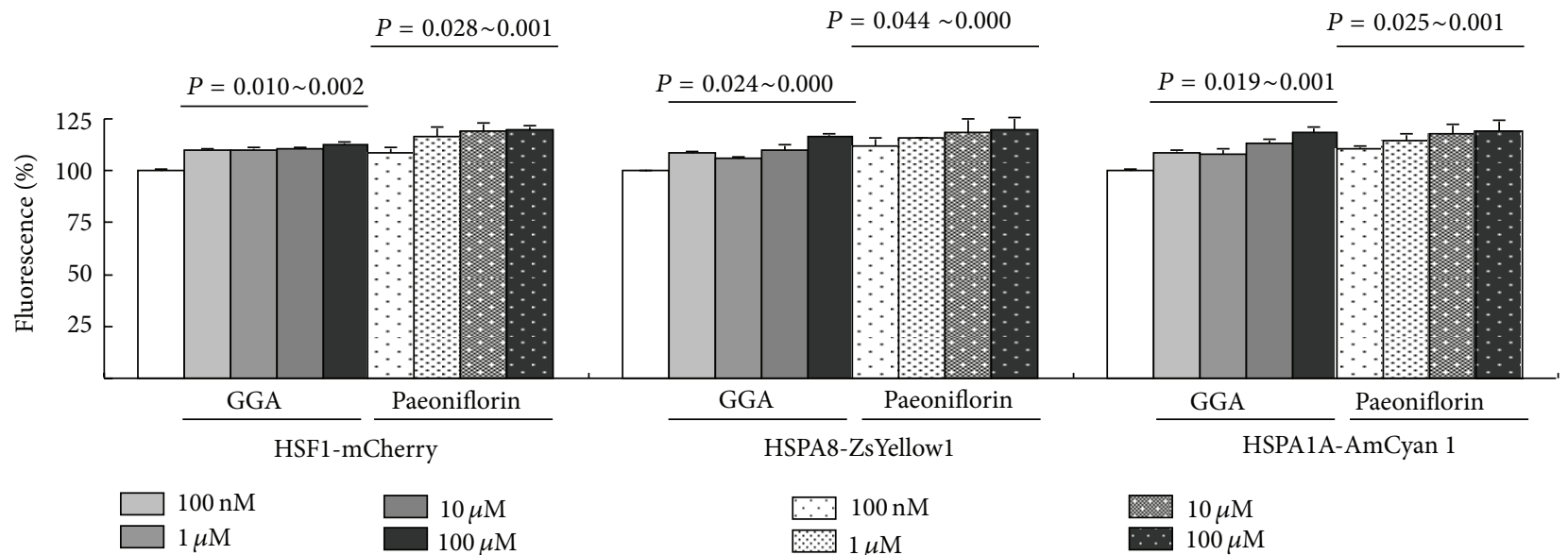

(c)
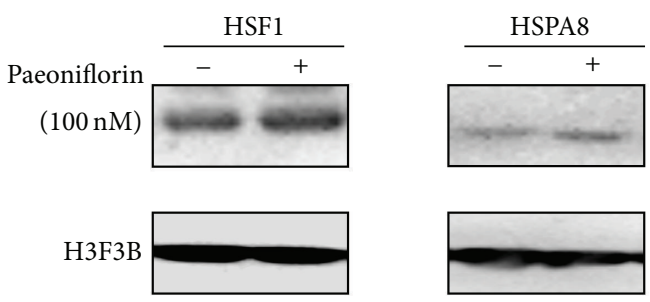

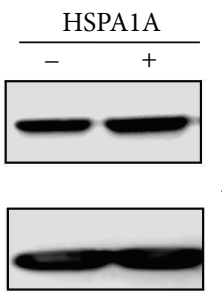

(d)

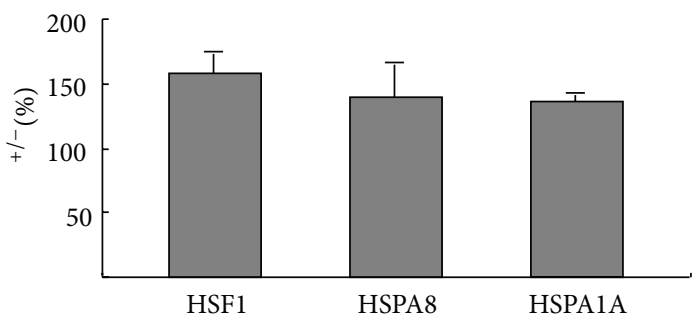

FIGURE 4: Enhancement of chaperone expression by paeoniflorin in 293 cells. (a) Triple fluorescent reporter plasmid with HSF1, HSPA8, and HSPA1A promoter fragments upstream of mCherry, ZsYellowl, and AmCyan1 fluorescent reporters, respectively. (b) Microscopic images of the triple fluorescent reporter cells. (c) Effect of GGA and paeoniflorin (100 nM 100 $\mu \mathrm{M})$ on HSF1, HSPA8, and HSPA1A reporters. To normalize, the fluorescence level in untreated cells is set as $100 \%$. Three independent experiments were performed with $P<0.05$ considered significant. (d) Representative western blot image of paeoniflorin-(100 nM) treated 293 cells for two days using HSF1, HSPA8, HSPA1A, and H3F3B antibodies. Levels of HSF1, HSPA8, HSPA1A, were normalized with a loading control (H3F3B). Data are expressed as the mean \pm SEM values from three independent experiments. 

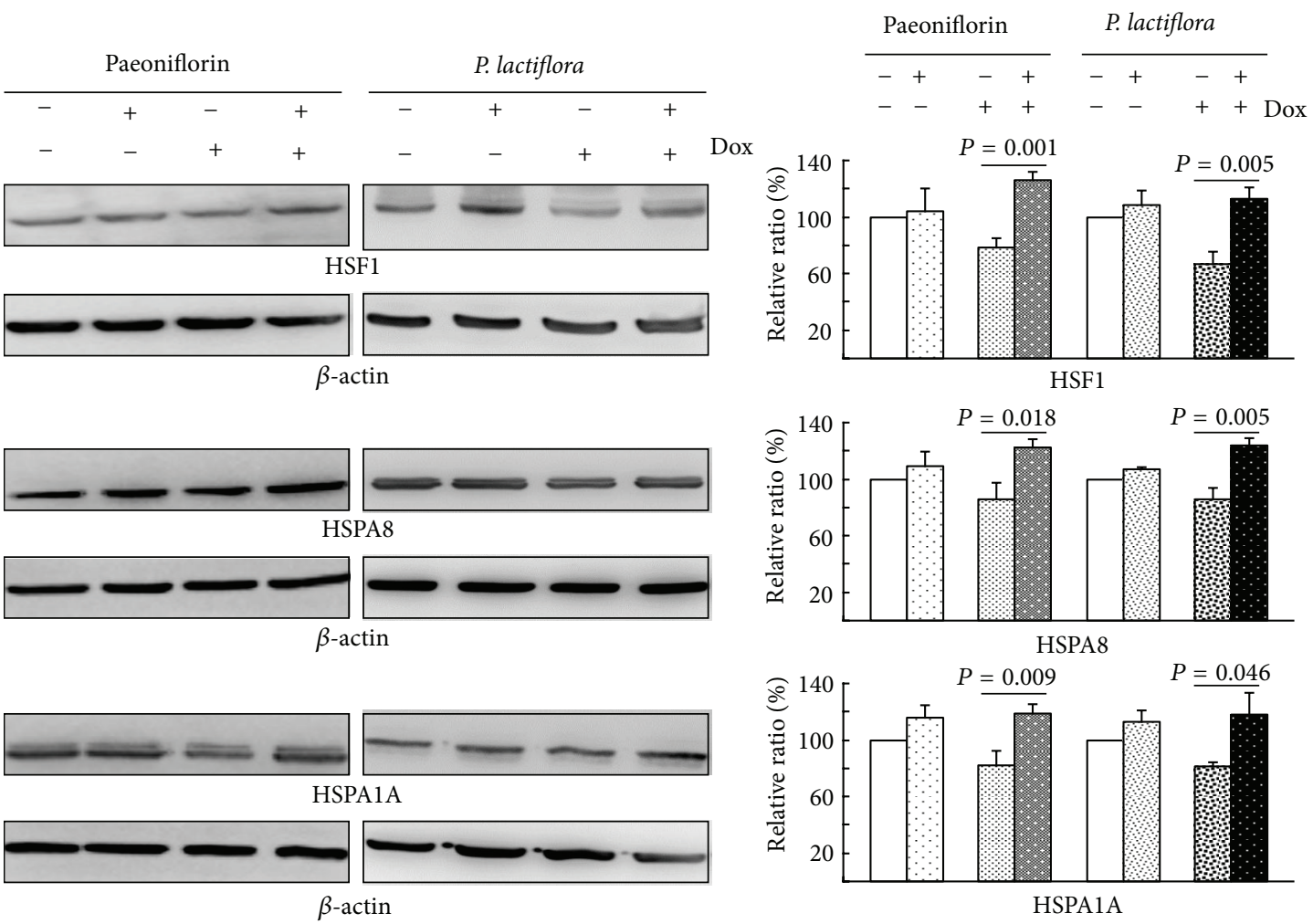

FIGURE 5: Enhancement of HSF1 and chaperone expression by paeoniflorin and the aqueous extract of P. lactiflora in ATXN3/Q A5 $^{-G F P ~} 293$ cells. Cells were pre-treated with paeoniflorin $(100 \mathrm{nM})$ or herb $(10 \mu \mathrm{g} / \mathrm{mL})$ for 8 hours and ATXN3/ ${ }_{75}$-GFP expression induced for 6 days. Relative HSF1, HSPA8, and HSPA1A expressions were analyzed by western blot analysis using $\beta$-actin as a loading control.

the chemical profile of aqueous extract was analyzed and quantified by full-spectrum analytic HPLC. Chromatographic patterns showed peaks in $230 \mathrm{~nm}$ corresponding to the retention time compatible with paeoniflorin, garlic acid and albiflorin (Figure 2(a)). The amounts of paeoniflorin, garlic acid, and albiflorin in aqueous extract of $P$. lactiflori were $2.27 \%, 0.30 \%$, and $0.73 \%$, respectively, corresponding to $47.33 \mathrm{mM}, 18.06 \mathrm{mM}$, and $15.16 \mathrm{mM}$, respectively, in $1 \mathrm{~g} / \mathrm{mL}$ aqueous extract (Figure 2(b)).

MTT assays were performed with human embryonic kidney 293 and human neuroblastoma SH-SY5Y cells after treatment with extract of $P$. lactiflora and the its three constituents, respectively, for $24 \mathrm{hr}$. The histone deacetylase inhibitor suberoylanilide suberoylanilide hydroxamic acid (SAHA) known to reduce SDS-insoluble polyQ aggregate [26] was included for comparison. The $\mathrm{IC}_{50}$ of the herb and compounds were calculated using the interpolation method. Both P. lactiflora extract and its constituents paeoniflorin and albiflorin had an $\mathrm{IC}_{50}$ higher than the highest concentration tested ( $>30 \mathrm{mg} / \mathrm{mL}$ for P. lactiflora and $>1 \mathrm{mM}$ for paeoniflorin and albiflorin), suggesting their very low cytotoxicity (Figure 2(c)).

3.3. P. lactiflori Extract and Paeoniflorin Reduce ATXN $3 / Q_{75}$ Aggregation on 293 Cell Model. To screen if herb/compounds potentially inhibit aggregation, we used $A T X N 3 / Q_{75}-G F P$ cells to examine aqueous extract of $P$. lactiflora and its constituents for their potentials to reduce the ATXN3/ $\mathrm{Q}_{75}$ aggregation. The experiment flow chart is shown in Figure 3(a) and representative fluorescence microscopy images of aggregation after treatment with paeoniflorin and the aqueous extract of $P$. lactiflora are shown in Figure 3(b). As a positive control, HDAC inhibitor SAHA reduced the ATXN3/ $\mathrm{Q}_{75}$ aggregation to $85 \%$ (at $100 \mathrm{nM}$ ) as compared to untreated cells (Figure 3(c)). While garlic acid did not display good aggregation-inhibitory potential $(90 \sim 95 \%$ at $100 \mathrm{nM} \sim 1 \mu \mathrm{M})$, P. lactiflora $(81 \sim 82 \%$ at $2 \sim 50 \mu \mathrm{g} / \mathrm{mL})$, paeoniflorin $(73 \%$ at $100 \mathrm{nM})$ and albiflorin $(78 \%$ at $5 \mu \mathrm{M})$ had greater aggregation reduction potential than SAHA (Figure 3(c)). The $\mathrm{IC}_{50}$ cytotoxicity/effective (reduced the ATXN3/ $\mathrm{Q}_{75}$ aggregation to $85 \%$ or lower) dose ratio of SAHA, paeoniflorin, albiflorin, and extract of $P$. lactiflora were $3800,>10000,>200$, and $>15000$, respectively. Considering $2 \mu \mathrm{g} / \mathrm{ml}$ of $P$. lactiflora extract contained $95 \mathrm{nM}$ paeoniflorin and $30 \mathrm{nM}$ albiflorin and tested greatest aggregation reduction potential of $100 \mathrm{nM}$ for paeoniflorin and $5 \mu \mathrm{M}$ for albiflorin, paeoniflorin was regarded as a major active component for the aggregation inhibition in P. lactiflora.

\subsection{Paeoniflorin Enhanced HSF1 and HSP70 Chaperone} Expression on 293 Cells. To screen the potential of herb/ compounds to enhance HSF1 and HSP70 chaperone expression, we established a triple fluorescent reporter 293 cell 

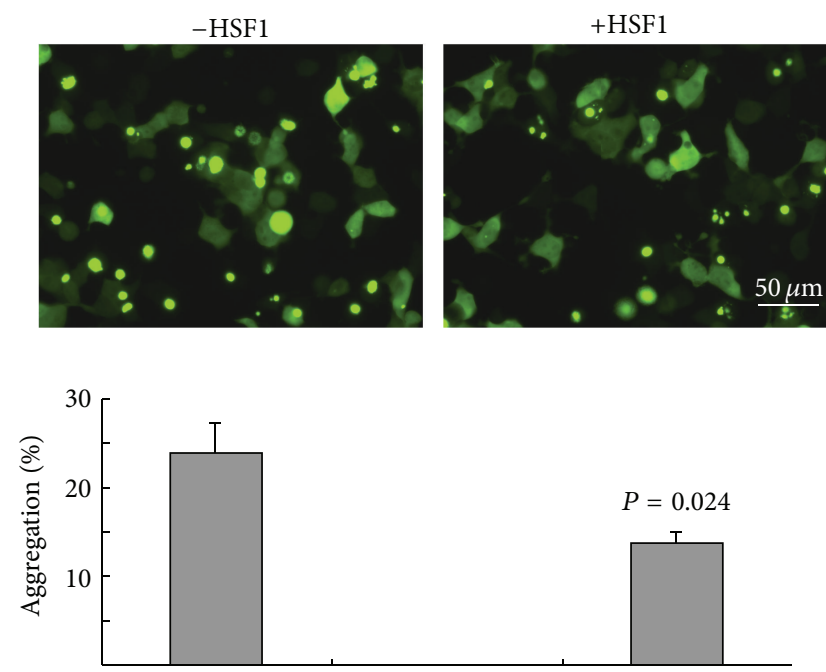

Figure 6: HSF1 overexpression in ATXN3/ ${ }_{75}$-GFP transient cell models. HEK-293T cells were co-transfected with plasmids encoding ATXN3/ $\mathrm{Q}_{75}$-GFP and plasmid with (+HSF1) or without (-HSF1) HSF1 cDNA. After 2 days, aggregation percentage was assessed by HCA system. (The scale bar $=50 \mu \mathrm{m}$ ). The percentage of aggregate formation counted among three random fields.

model with mCherry, ZsYellowl and AmCyan1 reporters downstream of HSF1, HSPA8 and HSPA1A promoters (Figure 4(a)). The cloned promoters effectively drove the expression of red, yellow, and blue fluorescent reporters (Figure 4(b)). As shown in Figure 4(c), treatment of GGA $(100 \mathrm{nM} \sim 100 \mu \mathrm{M})$, a potent HSP inducer, for one day significantly increased HSF1 (110\% 112\%, $P=0.010 \sim 0.002)$, HSPA8 $(106 \% \sim 116 \%, P=0.024 \sim 0.000)$ and HSPA1A $(108 \% \sim 118 \%$, $P=0.019 \sim 0.001)$ promoter activity. This was also true for paeoniflorin $(100 \mathrm{nM} \sim 100 \mu \mathrm{M})$ treatment, with $108 \% \sim 120 \%$ HSF1 $(P=0.028 \sim 0.001), 112 \% \sim 120 \%$ HSPA $8(P=0.044 \sim$ $0.000)$ and $110 \% \sim 119 \%$ HSPA1A $(P=0.025 \sim 0.001)$ promoter activities compared to no treament. The enhancement of paeoniflorin $(100 \mathrm{nM})$ on HSF1 (158\%, $P=0.027)$, HSPA8 $(140 \%, P=0.011)$ and HSPA1A $(137 \%, P=0.007)$ expression was confirmed by the Western blot in HEK-293 cells after two days treatment (Figure $4(\mathrm{~d})$ ).

\subsection{P. lactiflori Extract and Paeoniflorin Enhanced HSF1} and HSP70 Chaperone Expression on 293 ATXN3/Q ${ }_{75}$ Cell Model. To examine if paeoniflorin and P. lactiflora extract also up-regulated HSF1 and HSP70 chaperone expression in ATXN3/ ${ }_{75} 293$ cells, we compared the expression levels of HSF1, HSPA8 and HSPA1A between with and without paeoniflorin/P. lactiflora and/or Dox treatment. As shown in Figure 5, induced expression of ATXN3/Q $\mathrm{Q}_{75}$ for 6 days attenuated the expression of HSF1 (78\% 67\%), HSPA8 (86\%) and HSPA1A (82\%). This reduction can be rescued by the addition of paeoniflorin $(100 \mathrm{nM})$ or P. lactiflora $(10 \mu \mathrm{g} / \mathrm{mL})$, with significantly increased HSF1 $(113 \% \sim 126 \%, P=0.005 \sim$ $0.001)$, HSPA8 $(123 \% \sim 124 \%, P=0.018 \sim 0.005)$ and HSPA1A (118\% 119\%, $P=0.046 \sim 0.009)$ expression. These findings suggested that $P$. lactiflora and paeoniflorin up-regulated
HSF1 and HSP70 chaperon expression to reduce ATXN3/ $\mathrm{Q}_{75}$ aggregation in this cell model.

3.6. HSF1 Overexpression to Reduce ATXN3/Q ${ }_{75}$ Aggregation. To determine whether HSF1 could suppress aggregation of mutant ATXN3, we transiently co-expressed HSF1 with ATXN3/Q 75 in HEK-293T cells. As shown in Figure 6, with HSF1 co-transfection, visible aggregates significantly decreased in $\mathrm{ATXN} 3 / \mathrm{Q}_{75}$ cells $(13.8 \%$ versus $24.0 \%, P=$ 0.024).

3.7. P. lactiflori Extract and Paeoniflorin Reduced ATXN3/ $Q_{75}$ Aggregation on SH-SY5Y Cell Model. To test the aggregation reduction potential of $P$. lactiflori extract and paeoniflorin in neuronal cells, we constructed Flp-In SH-SY5Y cells with $\mathrm{N}$-terminal truncated ATXN3/Q $\mathrm{Q}_{14 \sim 75}$-GFP expression in an inducible fashion. GFP-tagged $40 \sim 57 \mathrm{kDa}$ ATXN3/Q $14 \sim 75$ protein in Dox-induced SH-SY5Y cells can be seen in Western blot (Figure 7(a)). Then we differentiated ATXN3/ $Q_{14 \sim 75}$ $\mathrm{SH}-\mathrm{SY} 5 \mathrm{Y}$ cells using retinoic acid and found that the induced $\mathrm{ATXN} 3 / \mathrm{Q}_{75}$ formed aggregates in $\sim 1 \%$ neuronal cells (Figure 7(b)). The treatment of paeoniflorin or P. lactiflora leaded to $21 \% \sim 16 \%$ of aggregation reduction $(P=0.013 \sim$ 0.035 ) in $A T X N 3 / Q_{75}$ expressed neuronal cells (Figure 7(c)). These results confirmed the aggregation-inhibitory effect of paeoniflorin and P. lactiflora in differentiated neurons.

\section{Discussion}

Although Chinese herbs have been reported to reduce pneumonia risk in elderly patients with dementia [27] and regarded as a potential treatment of Huntington's disease (HD) [28], the attempts to apply this alternative treatment in SCA are still few. Okabe et al. (2007) reported a patient with SCA6 was treated with a mixture of 18 medical herbs (modified Zhengan Xifeng Tang) and then the patient's ataxia was remarkably reduced [29]. However, the therapeutically effective compound(s) in this remedy remains unknown. In this study we identified the aqueous extract of $P$. lactiflora that reduced ATXN3-aggregates mainly via its active compound paeoniflorin (Figures 2 and 3). The reporter gene assay and Western blotting further indicated the aggregation-reduction effect of paeoniflorin was modulated by the up-regulation of HSF1 and its targets, HSPA8 and HSPA1A chaperone expressions (Figure 4).

P. lactiflora, with immunomodulatory and anti-inflammatory effects [30], has been widely used as a component of traditional Chinese prescriptions to relieve pain and to treat rheumatoid arthritis, systemic lupus erythromatosus, dysmenorrhea, hepatitis, muscle spasm, and fever with a long history. Its main bioactive component, paeoniflorin, possesses wide pharmacological effects in the nervous system. It has been reported to decrease the death of rat cortical cells while exposed to $\mathrm{H}_{2} \mathrm{O}_{2}$-induced oxidative stress [31]. Subcutaneous injection of paeoniflorin has shown functional protection in the 6-OHDA lesion rodent model of PD [16], 


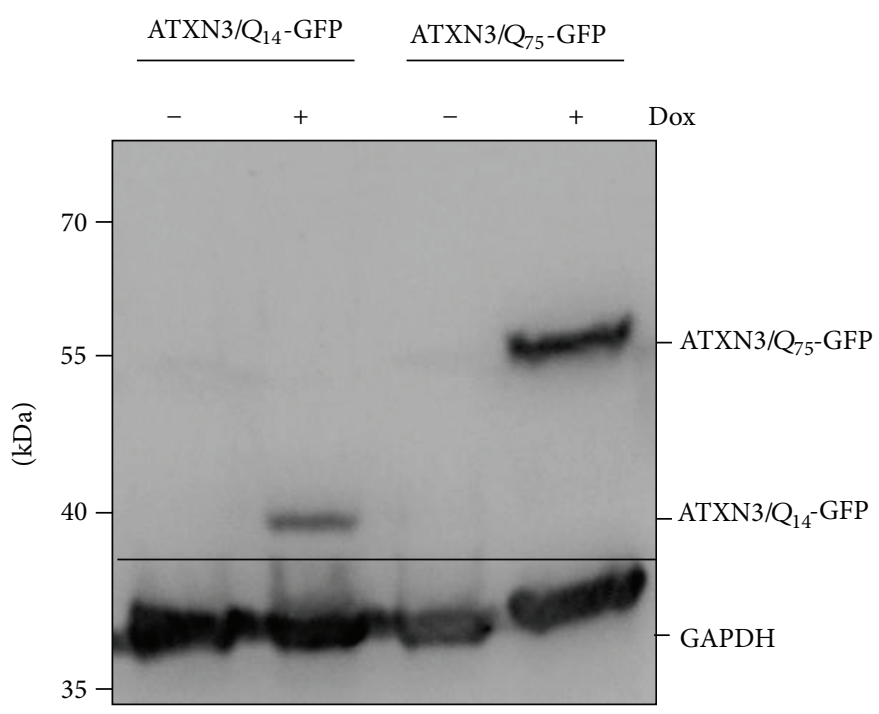

(a)

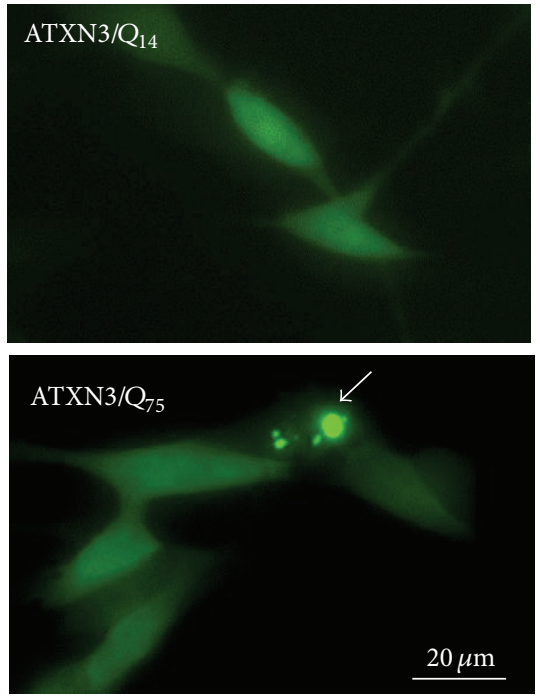

(b)

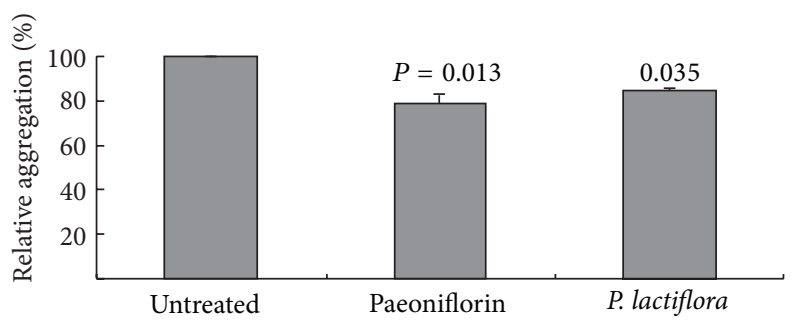

(c)

FIGURE 7: Reduction of aggregation by paeoniflorin and the aqueous extract of $P$. lactiflora in ATXN3/ ${ }_{75}-$ GFP SH-SY5Y cells. (a) Western blot analysis using GFP and GAPDH antibodies after two days induction (+Dox). (b) Microscopic images of differentiated SH-SY5Y cells expressing ATXN3/ $\mathrm{Q}_{14 / 75}$-GFP for 6 days (the scale bar $=20 \mu \mathrm{m}$ ). (c) Relative aggregation after treatment with paeoniflorin (100 $\mathrm{nM}$ ) or the aqueous extract of $P$. lactiflora $(10 \mu \mathrm{g} / \mathrm{mL})$ for 6 days. To normalize, the relative aggregation level in untreated cells is set as $100 \%$.

and reduce the MPTP-induced toxicity by activation of the adenosine $\mathrm{Al}$ receptor to inhibit neuroinflammation [15]. In $\mathrm{A} \beta_{(1-42)}$-injected $\mathrm{AD}$ rat model, paeoniflorin attenuates the neurotoxicity and cognitive decline by regulating calcium homeostasis and ameliorating oxidative stress [17]. Our results demonstrate both extract of $P$. lactiflora and paeoniflorin up-regulating HSF1 and HSP70 expressions to inhibit aggregate formation in ATXN3/ $\mathrm{Q}_{75} 293$ cells (Figure 5). This aggregation-inhibitory effect can also be seen in neuronal differentiated SH-SY5Y cells expressing ATXN3/ $\mathrm{Q}_{75}$ (Figure 7), providing a novel mechanism of $P$. lactiflora and paeoniflorin to slow down the neurodegenerative process by inducing the expression of heat shock proteins.

Peoniflorin was the first reported active component of herbal medicines to induce expression of heat shock proteins in HeLa, IMR-32, and normal rat kidney cells [32]. Upon stress, HSF1 is released from the chaperone complex, selftrimerizes, and then is transported into the nucleus as a transcription factor. It activates chaperones, which play an important role in preventing unwanted protein aggregation. Overexpression of HSF1 significantly improved the life span of R6/2 Huntington's disease mouse [33]. Up-regulation of chaperone expression by HSF1 and its activating compounds 17-(allylamino)-17-demethoxygeldanamycin (17-AAG) demonstrate a strong inhibitory effect on HD aggregate formation [34]. We also showed aggregation-inhibitory effect of HSF1 overexpression in 293 cells expressing ATXN3/ $\mathrm{Q}_{75}$ (Figure 6). Overexpression of its downstream target gene HSPA1A suppresses polyQ-mediated neurodegeneration in Drosophila and mouse models [35, 36]. Reduced expression of HSPA8, another target genes of HSF1 [37], has been shown in the 293 cells overexpressing expanded TBP-Q ${ }_{61}[38]$ and SCA17 lymphoblastoid cells [39]. The therapeutic potential of $P$. lactiflora and paeoniflorin in the treatment of SCA is strongly supported by the up-regulated HSF1 and HSP70 expressions.

In conclusion, our in vitro study provides strong evidence that P. lactiflora and paeoniflorin could be novel therapeutics for SCA3 and other polyQ diseases. Future application of P. lactiflora and paeoniflorin to SCA animal models would solidify their effects on aggregation reduction and disease improvement. Since the pathogenesis of the polyQ diseases is not completely clear and effective treatment is not available, our cell models are extremely valuable for identifying potential therapeutic targets in polyQ diseases. A systemic highthroughput screening of herbal and chemical compounds using ATXN3/ $\mathrm{Q}_{75} 293$ cell model is undergoing. 


\section{Conflict of Interests}

The authors declare that there is no conflict of interests.

\section{Acknowledgments}

This work was supported by Grants NSC100-2325-B-003001 and NSC101-2325-B-003 -002 from the National Science Council, Executive Yuan, NTNU100-D-02 from National Taiwan Normal University, Taipei, and Aim for the Top University Project-NTNU, Taiwan. K.-H. Chang, W.-L. Chen, and L.-C. Lee equally contributed to this work.

\section{References}

[1] A. Matilla-Dueñas, M. Corral-Juan, V. Volpini, and I. Sanchez, "The spinocerebellar ataxias: clinical aspects and molecular genetics," Advances in Experimental Medicine and Biology, vol. 724, pp. 351-374, 2012.

[2] Y. Kawaguchi, T. Okamoto, M. Taniwaki et al., "CAG expansions in a novel gene for Machado-Joseph disease at chromosome 14q32.1," Nature Genetics, vol. 8, no. 3, pp. 221-228, 1994.

[3] H. T. Orr, M. Y. Chung, S. Banfi et al., "Expansion of an unstable trinucleotide CAG repeat in spinocerebellar ataxia type 1," Nature Genetics, vol. 4, no. 3, pp. 221-226, 1993.

[4] S. M. Pulst, A. Nechiporuk, T. Nechiporuk et al., "Moderate expansion of a normally biallelic trinucleotide repeat in spinooerebellar ataxia type," Nature Genetics, vol. 14, no. 3, pp. 269-276, 1996.

[5] O. Zhuchenko, J. Bailey, P. Bonnen et al., "Autosomal dominant cerebellar ataxia (SCA6) associated with small polyglutamine expansions in the $\alpha_{(1 A)}$-voltage-dependent calcium channel," Nature Genetics, vol. 15, no. 1, pp. 62-69, 1997.

[6] G. David, N. Abbas, G. Stevanin et al., "Cloning of the SCA7 gene reveals a highly unstable CAG repeat expansion," Nature Genetics, vol. 17, no. 1, pp. 65-70, 1997.

[7] R. Koide, S. Kobayashi, T. Shimohata et al., "A neurological disease caused by an expanded CAG trinucleotide repeat in the TATA-binding protein gene: a new polyglutamine disease?" Human Molecular Genetics, vol. 8, no. 11, pp. 2047-2053, 1999.

[8] R. Koide, T. Ikeuchi, O. Onodera et al., "Unstable expansion of CAG repeat in hereditary dentatorubral-pallidoluysian atrophy (DRPLA)," Nature Genetics, vol. 6, no. 1, pp. 9-13, 1994.

[9] H. Y. Zoghbi and H. T. Orr, "Polyglutamine diseases: protein cleavage and aggregation," Current Opinion in Neurobiology, vol. 9, no. 5, pp. 566-570, 1999.

[10] J. R. Gatchel and H. Y. Zoghbi, "Diseases of unstable repeat expansion: mechanisms and common principles," Nature Reviews Genetics, vol. 6, no. 10, pp. 743-755, 2005.

[11] A. Solans, A. Zambrano, M. Rodríguez, and A. Barrientos, "Cytotoxicity of a mutant huntingtin fragment in yeast involves early alterations in mitochondrial OXPHOS complexes II and III," Human Molecular Genetics, vol. 15, no. 20, pp. 3063-3081, 2006.

[12] E. J. Bennett, T. A. Shaler, B. Woodman et al., "Global changes to the ubiquitin system in Huntington's disease," Nature, vol. 448, no. 7154, pp. 704-708, 2007.

[13] S. M. Chafekar and M. L. Duennwald, "Impaired heat shock response in cells expressing full-length polyglutamineexpanded Huntingtin," PLOS ONE, vol. 7, no. 5, Article ID e37929, 2012.
[14] T. T. Ou, C. H. Wu, J. D. Hsu, C. C. Chyau, H. J. Lee, and C. J. Wang, "Paeonia lactiflora Pall inhibits bladder cancer growth involving phosphorylation of Chk2 in vitro and in vivo," Journal of Ethnopharmacology, vol. 135, no. 1, pp. 162-172, 2011.

[15] H. Q. Liu, W. Y. Zhang, X. T. Luo, Y. Ye, and X. Z. Zhu, "Paeoniflorin attenuates neuroinflammation and dopaminergic neurodegeneration in the MPTP model of Parkinson's disease by activation of adenosine A1 receptor," British Journal of Pharmacology, vol. 148, no. 3, pp. 314-325, 2006.

[16] D. Z. Liu, J. Zhu, D. Z. Jin et al., "Behavioral recovery following sub-chronic paeoniflorin administration in the striatal 6OHDA lesion rodent model of Parkinson's disease," Journal of Ethnopharmacology, vol. 112, no. 2, pp. 327-332, 2007.

[17] S. Z. Zhong, Q. H. Ge, Q. Li, R. Qu, and S. P. Ma, "Peoniflorin attentuates $\mathrm{A} \beta_{(1-42)}$-mediated neurotoxicity by regulating calcium homeostasis and ameliorating oxidative stress in hippocampus of rats," Journal of the Neurological Sciences, vol. 280, no. 1-2, pp. 71-78, 2009.

[18] M. H. Jeon, H. J. Kwon, J. S. Jeong, Y. M. Lee, and S. P. Hong, "Detection of albiflorin and paeoniflorin in Paeoniae Radix by reversed-phase high-performance liquid chromatography with pulsed amperometric detection," Journal of Chromatography A, vol. 1216, no. 21, pp. 4568-4573, 2009.

[19] S. L. Li, J. Z. Song, F. F. K. Choi et al., "Chemical profiling of Radix Paeoniae evaluated by ultra-performance liquid chromatography/photo-diode-array/quadrupole time-of-flight mass spectrometry," Journal of Pharmaceutical and Biomedical Analysis, vol. 49, no. 2, pp. 253-266, 2009.

[20] Y. Zhang, S. Koushik, R. Dai, and N. F. Mivechi, "Structural organization and promoter analysis of murine heat shock transcription factor-1 gene," Journal of Biological Chemistry, vol. 273, no. 49, pp. 32514-32521, 1998.

[21] M. He, H. Guo, X. Yang et al., "Genetic variations in HSPA8 gene associated with coronary heart disease risk in a Chinese population," PloS One, vol. 5, no. 3, p. e9684, 2010.

[22] Y.-R. Wu, C.-K. Wang, C.-M. Chen et al., "Analysis of heatshock protein 70 gene polymorphisms and the risk of Parkinson's disease," Human Genetics, vol. 114, no. 3, pp. 236-241, 2004.

[23] L.-C. Lee, C.-M. Chen, H.-C. Wang et al., "Role of the CCAATbinding protein NFY in SCA17 pathogenesis," PLoS One, vol. 7, no. 4, Article ID e35302, 2012.

[24] S. Flis and J. Spławiński, "Inhibitory effects of 5-fluorouracil and oxaliplatin on human colorectal cancer cell survival are synergistically enhanced by sulindac sulfide," Anticancer Research, vol. 29, no. 1, pp. 435-441, 2009.

[25] A. Haacke, S. A. Broadley, R. Boteva, N. Tzvetkov, F. U. Hartl, and P. Breuer, "Proteolytic cleavage of polyglutamine-expanded ataxin-3 is critical for aggregation and sequestration of nonexpanded ataxin-3," Human Molecular Genetics, vol. 15, no. 4, pp. 555-568, 2006.

[26] M. Mielcarek, C. L. Benn, S. A. Franklin et al., "SAHA decreases HDAC 2 and 4 levels in vivo and improves molecular phenotypes in the R6/2 mouse model of Huntington's disease," PLoS One, vol. 6, no. 11, Article ID e27746, 2011.

[27] K. Iwasaki, S. Kato, Y. Monma et al., "A pilot study of Banxia Houpu Tang, a traditional Chinese medicine, for reducing pneumonia risk in older adults with dementia," Journal of the American Geriatrics Society, vol. 55, no. 12, pp. 2035-2040, 2007.

[28] T. Satoh, T. Takahashi, K. Iwasaki et al., "Traditional Chinese medicine on four patients with Huntington's disease," Movement Disorders, vol. 24, no. 3, pp. 453-455, 2009. 
[29] T. Okabe, M. Fujisawa, T. Sekiya, Y. Ichikawa, and J. Goto, "Successful treatment of spinocerebellar ataxia 6 with medicinal herbs," Geriatrics \& Gerontology International, vol. 7, no. 2, pp. 195-197, 2007.

[30] D.-Y. He and S.-M. Dai, "Anti-inflammatory and immunomodulatory effects of Paeonia lactiflora Pall., a traditional Chinese herbal medicine," Frontiers in Pharmacology, vol. 2, article 10, 2011.

[31] S. H. Kim, M. K. Lee, K. Y. Lee, S. H. Sung, J. Kim, and Y. C. Kim, "Chemical constituents isolated from Paeonia lactiflora roots and their neuroprotective activity against oxidative stress in vitro," Journal of Enzyme Inhibition and Medicinal Chemistry, vol. 24, no. 5, pp. 1138-1140, 2009.

[32] D. Yan, K. Saito, Y. Ohmi, N. Fujie, and K. Ohtsuka, "Paeoniflorin, a novel heat shock protein-inducing compound," Cell Stress and Chaperones, vol. 9, no. 4, pp. 378-389, 2004.

[33] M. Fujimoto, E. Takaki, T. Hayashi et al., "Active HSF1 significantly suppresses polyglutamine aggregate formation in cellular and mouse models," Journal of Biological Chemistry, vol. 280, no. 41, pp. 34908-34916, 2005.

[34] N. Fujikake, Y. Nagai, H. A. Popiel, Y. Okamoto, M. Yamaguchi, and T. Toda, "Heat shock transcription factor 1-activating compounds suppress polyglutamine-induced neurodegeneration through induction of multiple molecular chaperones," Journal of Biological Chemistry, vol. 283, no. 38, pp. 26188-26197, 2008.

[35] J. M. Warrick, H. Y. E. Chan, G. L. Gray-Board, Y. Chai, H. L. Paulson, and N. M. Bonini, "Suppression of polyglutaminemediated neurodegeneration in Drosophila by the molecular chaperone HSP70," Nature Genetics, vol. 23, no. 4, pp. 425-428, 1999.

[36] C. J. Cummings, Y. Sun, P. Opal et al., "Over-expression of inducible HSP70 chaperone suppresses neuropathology and improves motor function in SCA1 mice," Human Molecular Genetics, vol. 10, no. 14, pp. 1511-1518, 2001.

[37] S. A. Kim, J. H. Yoon, D. K. Kim, S. G. Kim, and S. G. Ahn, "CHIP interacts with heat shock factor 1 during heat stress," FEBS Letters, vol. 579, no. 29, pp. 6559-6563, 2005.

[38] L. C. Lee, C. M. Chen, F. L. Chen et al., "Altered expression of HSPA5, HSPA8 and PARK7 in spinocerebellar ataxia type 17 identified by 2-dimensional fluorescence difference in gel electrophoresis," Clinica Chimica Acta, vol. 400, no. 1-2, pp. 5662, 2009.

[39] C. M. Chen, L. C. Lee, B. W. Soong et al., "SCA17 repeat expansion: mildly expanded CAG/CAA repeat alleles in neurological disorders and the functional implications," Clinica Chimica Acta, vol. 411, no. 5-6, pp. 375-380, 2010. 


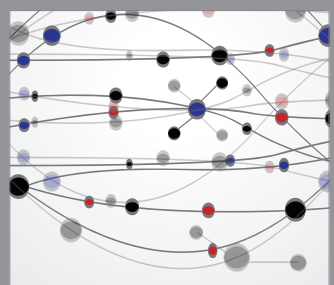

The Scientific World Journal
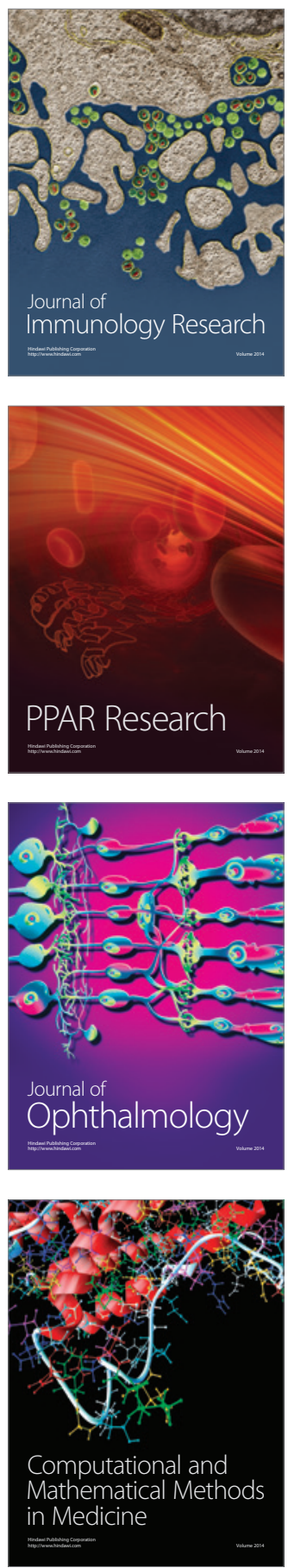

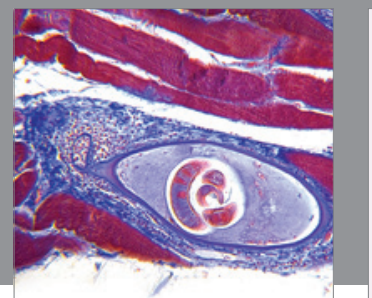

Gastroenterology

Research and Practice
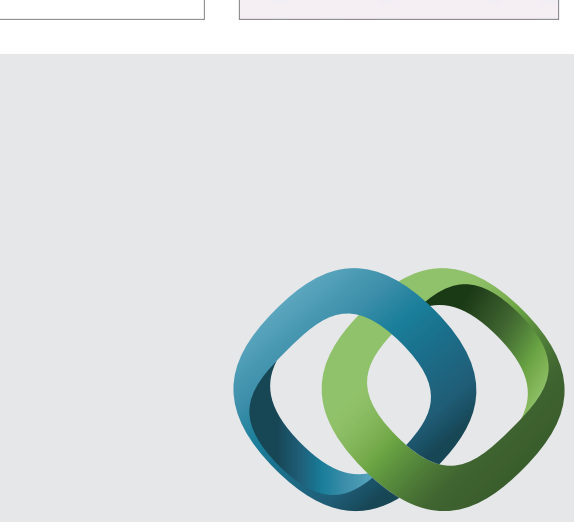

\section{Hindawi}

Submit your manuscripts at

http://www.hindawi.com
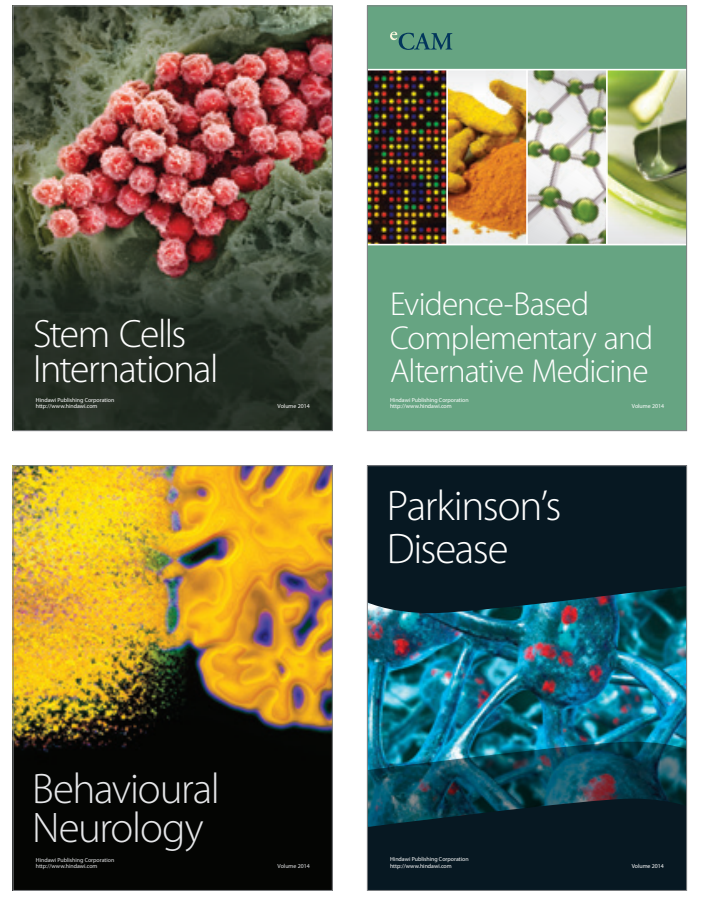
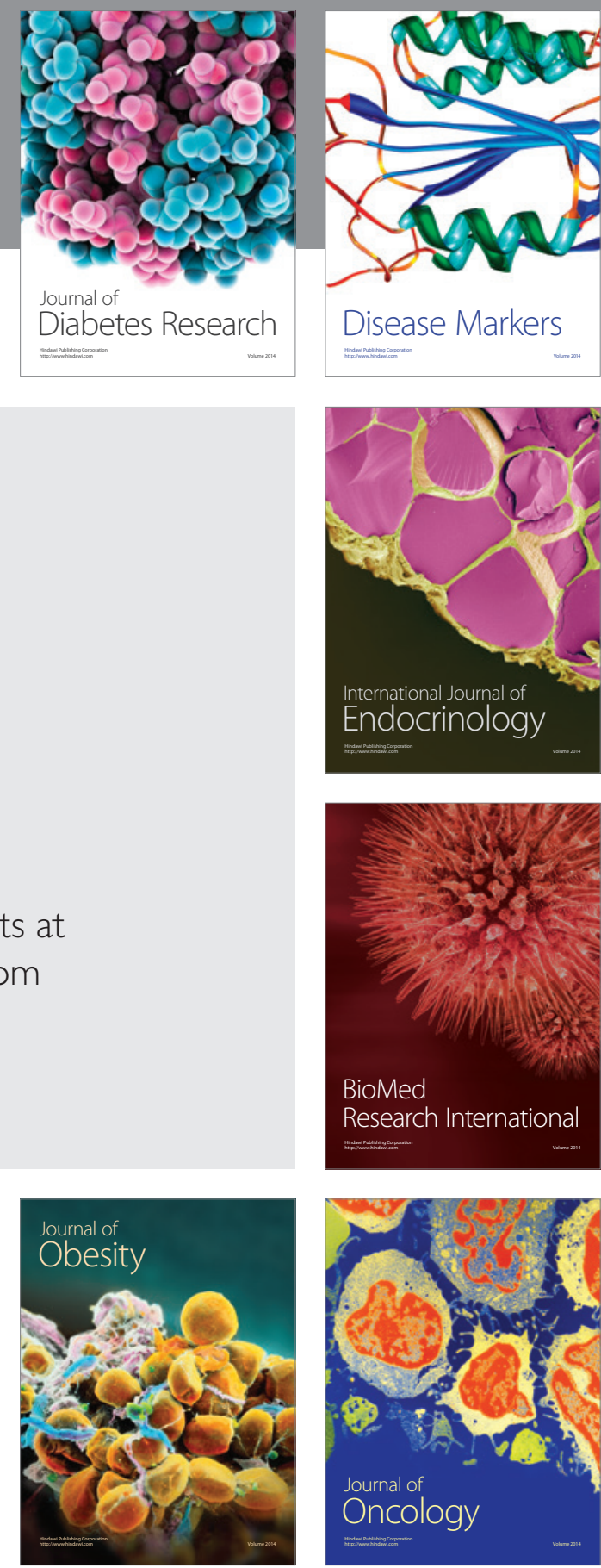

Disease Markers
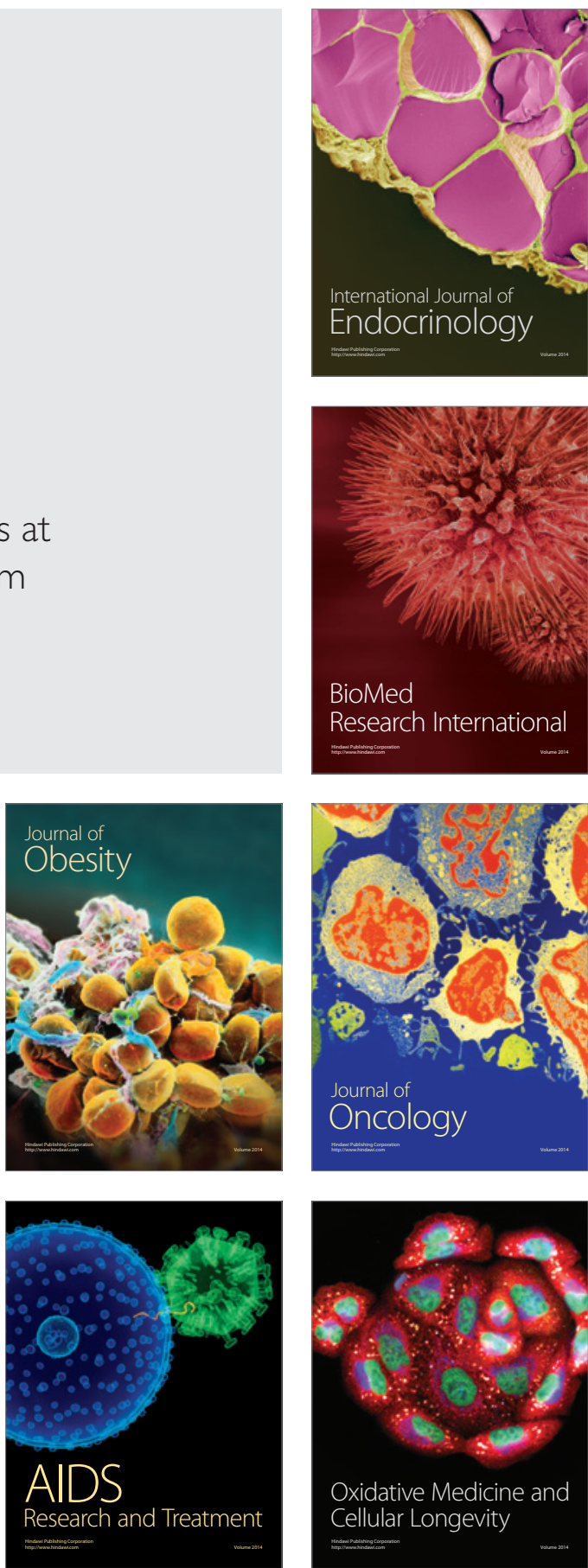